\title{
EXPERIENCIAS PARA EL MANEJO INTEGRADO DE CERCOSPORA NICOTIANAE ELLIS \& EVERH EN TABACO NEGRO, LOCALIDAD DE JUAN GUERRA. TARAPOTO. PERÚ
}

\author{
EXPERIENCES FOR THE MANAGEMENT OF CERCOSPORA \\ NICOTIANAE ELLIS \& EVERH IN BLACK TOBACCO-TOWN \\ OF JUAN GUERRA, TARAPOTO-PERU
}

\author{
Alberto Julca Otiniano ${ }^{1}$; Noemí Julca Vera ${ }^{1}$; Raúl Blas-Sevillano ${ }^{1}$; Segundo Bello Amez ${ }^{2}$; \\ Ruth Carhuallanqui Pérez ${ }^{1}$; Reynaldo Crespo Costa ${ }^{1}$
}

\section{RESUMEN}

\begin{abstract}
En la localidad de Juan Guerra (Tarapoto, Perú) se realizaron diversos estudios que permitieran diseñar en el futuro un programa de manejo integrado de la "Cercosporiosis" causada por Cercospora nicotianae en tabaco negro para puros (habanos).

Se determinó que la severidad y la incidencia de la enfermedad están altamente correlacionadas. La severidad aumentó con la edad de la planta de tabaco y de las dos variedades evaluadas la HN2 fue la que presentó la menor severidad. La "Cercosporiosis" fue más importante en la época lluviosa, época en la que se registró la mayor severidad.

También se encontró que la densidad de plantación no afectó significativamente la severidad de la enfermedad y tampoco hubo efecto significativo de los niveles de fertilización mineral estudiados. La severidad fue significativamente menor cuando el control de brotes axilares se realizó manualmente y la aplicación de fungicidas controló significativamente la enfermedad. Los mejores resultados estuvieron asociados con la mezcla de fungicidas de contacto y sistémicos.
\end{abstract}

Palabras clave: Densidad, fertilización, control químico, escala, modelos regresión.

\begin{abstract}
It was determined that the severity and the incidence of the disease are highly correlated. The severity, increased with the age of the tobacco plant. When comparing the two evaluated varieties the HN2 was the one that showed the less severity. The "Cercosporiosis" was more important in the rainy season in which the greatest severity was recorded.

It was also found that the plantation density didn't affect significantly the severity of the disease. Neither there was a significant effect of the mineral fertilization levels under study. The severity was significantly smaller when the control of growth of axillary buds was carried out manually and the application of fungicides controlled the disease significantly. The best results were associated with the mixture of contact and systemic fungicides.
\end{abstract}

Key words: Density, fertilization, chemical control, regression model.

\section{INTRODUCCIÓN}

En la localidad de Juan Guerra (Tarapoto, Perú), se cultiva tabaco negro para puros (habanos). La rentabilidad del cultivo depende principalmente de la capacidad que tengan los agricultores de producir hojas limpias que puedan ser usadas como capa o capote; éstas tienen un precio significativamente mayor comparadas con las hojas que se usan para tripa o relleno (Echeandía y Ocampo, 2002). Dentro de este contexto, el principal problema fitosanitario es la "Cercosporiosis" (también llamada "ojo de sapo" u "ojo de rana") causada por Cercospora nicotianae Ellis \& Everh (Figura 1), esta es una

\footnotetext{
Universidad Nacional Agraria La Molina, Depto. Fitotecnia. Aptdo. 12056. La Molina. Lima.

2 Agrícola Italia S.R.L. Jr. Apuriac. 248. San Ramón. Chanchamayo. E-mail: ajo@ lamolina.edu.pe
}

Fecha de Recepción: 13 Abril 2007

Fecha de Aceptación: 06 Agosto 2007 
enfermedad muy antigua y fue reportada por primera vez en Carolina del Norte (USA) por Ellis y Everh en 1893. Es más frecuente entre los $35^{\circ} \mathrm{LN}$ y $35^{\circ} \mathrm{LS}$, zona donde se encuentran los principales países productores de tabaco en el mundo, pero las pérdidas son más frecuentes en los países tropicales (APS, 1991).

Las experiencias para el control de C. nicotianae han estado dirigidas principalmente al uso de fungicidas químicos, con resultados diversos. Sin embargo, la tendencia actual es a disminuir el uso de éstos, lo que hace necesario desarrollar nuevos conocimientos que permitan el manejo integrado de esta enfermedad porque en el trópico los agroecosistemas son más frágiles a los cambios internos, como es el caso de Tarapoto. Según Cisneros (1980), el manejo integrado de plagas (MIP) es un sistema orientado a mantener las plagas de un cultivo en niveles que no causen daño económico, utilizando preferentemente los factores naturales adversos al desarrollo de plagas y sólo recurre al uso de pesticidas como medida de emergencia. El CATIE de Costa Rica lo define como un proceso de toma de decisiones sobre prácticas a usar, basado en observaciones sistemáticas y razonamiento ecológico sobre el cultivo, las plagas y el control natural, para mantener las pérdidas por plagas en niveles aceptables, con costos razonables y con un impacto mínimo sobre el medio ambiente y la salud humana.

Del párrafo anterior se desprende que para el diseño de un programa de MIP es necesario desarrollar experiencias específicas para la localidad en estudio. Éstas deben comprender la evaluación de daños y los diferentes métodos de control. En este documento se presentan los resultados de los diferentes trabajos de investigación realizados para el caso de Cercospora nicotianae en la localidad de
Juan Guerra (Tarapoto, Perú) y financiados por la empresa Tabacos del Perú S.A. (TAPESA).

Los objetivos fueron conocer la relación entre la severidad y la incidencia de la enfermedad, entre la severidad y la edad de la planta de tabaco, la respuesta de dos variedades comerciales a la enfermedad y el efecto de la época del cultivo. También se determinó el efecto de la densidad de plantación, la fertilización, el control de los brotes axilares y el control químico de la "Cercosporiosis".

\section{MATERIALES Y MÉTODOS}

Los trabajos se realizaron en el Fundo "Pucayacu" de la Universidad Nacional Agraria La Molina (UNALM), ubicado a orillas del río Cumbaza, en el distrito de Juan Guerra, provincia de Tarapoto, departamento de San Martín, selva nororiental del Perú. El área total cultivada con tabaco negro fue de 14 ha (12 ha con la var. HN2 y 2 ha con la var. HN1), bajo riego financiada y manejada por TAPESA, la que trabajaba de manera asociada con pequeños agricultores.

La zona tiene un clima tropical húmedo, con una precipitación promedio anual de $1352 \mathrm{~mm}$ distribuidos irregularmente a lo largo del año, la temperatura promedio anual es de $23,5^{\circ} \mathrm{C}$, con una máxima de $31,4^{\circ} \mathrm{C}$ y mínima de $15,7^{\circ} \mathrm{C}$; la humedad relativa se mantiene entre $75 \%$ a $85 \%$. El suelo es de naturaleza aluvial, de textura franco arcilloso, color pardo y $\mathrm{pH}$ de 7,7 ; la topografía es casi plana y tiene entre $2,0 \%$ y $3,0 \%$ de materia orgánica.

Para el manejo agronómico del cultivo de tabaco se consideraron las recomendaciones dadas para la producción comercial del tabaco negro en la zona de Juan Guerra que considera una densidad de 33.300 plantas/ha y una fórmula de fertilización

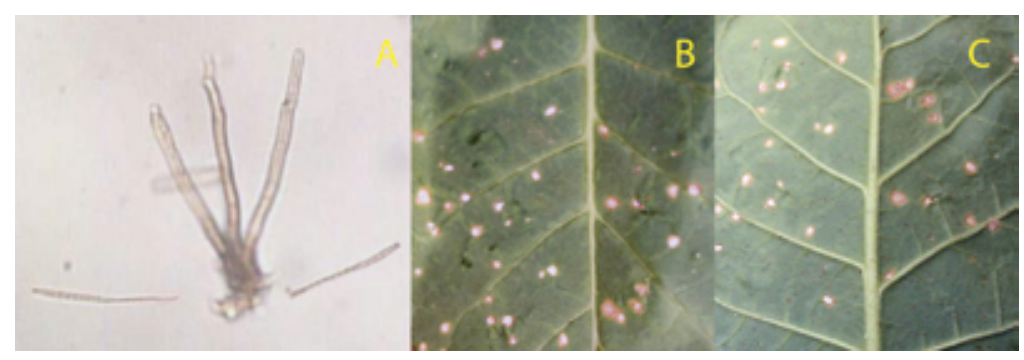

Figura 1. La "cercosporiosis", causada por Cercospora nicotianae (A) síntomas en haz (B) y envés (C) en tabaco var. HN2. Tarapoto. Perú. 
(kg/ha) de $90 \mathrm{~N}-90 \mathrm{P}_{2} \mathrm{O} 5-90 \mathrm{~K}_{2} \mathrm{O}-30 \mathrm{MgO}$, usando el fertilizante compuesto 12-12-12-04 de $\mathrm{N}-\mathrm{P}_{2} \mathrm{O} 5-\mathrm{K}_{2} \mathrm{O}$ y $\mathrm{MgO}$, de uso común en las zonas productoras de tabaco en el Perú.

La cosecha se realizó por el sistema de hoja por hoja y empezó 45 días después del trasplante, momento en que las primeras hojas basales reunían las condiciones adecuadas de madurez (Crespo y Julca, 2005). En total se realizaron cinco cortes, con un intervalo de aproximadamente una semana entre un corte y otro; en cada corte se arrancaron de 3 a 4 hojas/planta.

Las hojas cosechadas se trasladaron a los galpones de secado ("caney"), donde se pesaron (peso fresco) y luego se realizó el "encujado", que consiste en coser las hojas en un palo denominado cuje (Crespo y Julca, 2005). Los cujes se colgaron en el "caney" para que las hojas se secaran a temperatura ambiente en un proceso que duró aproximadamente 40 días. Pasado este tiempo, se bajaron los cujes, se sacaron las hojas completamente secas y fueron pesadas (peso seco).

\section{ESTUDIOS REALIZADOS:}

\section{Análisis de la metodología de evaluación}

Con el objetivo de conocer la relación entre la incidencia y la severidad de la enfermedad en un campo de la var. HN2, se delimitó un área experimental de aproximadamente una hectárea y se tomaron 200 plantas al azar en las que se midieron ambas variables durante el periodo del cultivo. La incidencia de la enfermedad se calculó con la siguiente fórmula:

Incidencia $=($ Nro. plantas enfermas $/$ Nro. total de plantas evaluadas) x 100
Para medir la severidad se usó una metodología propuesta por Julca y Crespo (1998). Ésta considera una escala gráfica de cinco grados (0-4), pero la media que se obtiene al evaluar cada hoja individualmente se corrige multiplicando dicho valor por un factor de corrección que varía según la ubicación de la hoja en la planta, denominándose a esta Severidad por planta $\left(\mathrm{S}_{\mathrm{p}}\right)$.

Los factores de corrección se obtuvieron dividiendo el número de hojas bajeras, medias y de corona, sobre el número total de hojas por planta. Para el caso de las hojas bajeras $\left(S_{b}\right)$ fue $4 / 18=$ 0,22 ; para hojas medias $\left(\mathrm{S}_{\mathrm{m}}\right): 10 / 18=0,56$ y para hojas de corona $\left(S_{c}\right): 4 / 18=0,22$. Finalmente la $\mathrm{S}_{\mathrm{p}}$ se calcula con la siguiente fórmula:

$$
S_{p}=S_{b}(0,22)+S_{m}(0,56)+S_{c}(0,22)
$$

Los datos fueron sometidos a un Análisis de Regresión Lineal simple.

\section{Efecto de la época del cultivo}

Con el objetivo de conocer el efecto de la época de cultivo en la enfermedad en un campo de la var. HN2 sembrada en cada época, se delimitó un área experimental de aproximadamente una hectárea y en cada oportunidad se tomaron 200 plantas al azar en las que se midió la severidad por planta (Sp). La época lluviosa (marzo-mayo) se caracteriza por tener una temperatura de $26,3{ }^{\circ} \mathrm{C}$, una humedad relativa de 78,5\% y una precipitación de 394,2 $\mathrm{mm}$; mientras que la época seca (junio-agosto) presenta una temperatura de $25,6{ }^{\circ} \mathrm{C}$, una humedad relativa de $76,9 \%$ y una precipitación de 204,5 mm. Los datos corresponden a un promedio de nueve años, según el Servicio de Meteorología e Hidrología del Perú (SENAMHI).
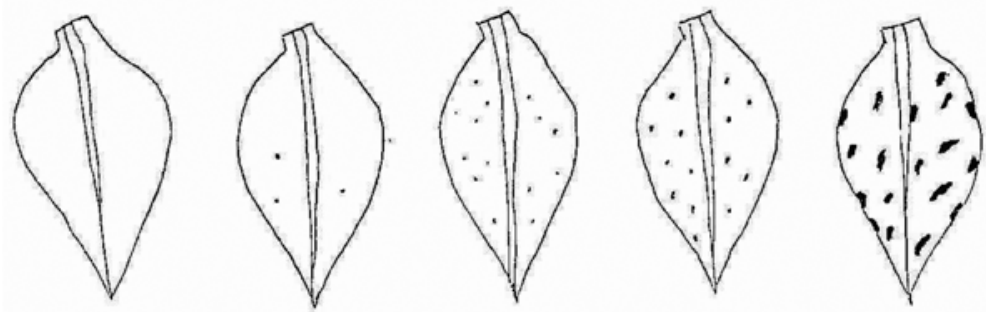

Figura 2. Escala gráfica para evaluar la severidad de Cercospora nicotianae en hojas de tabaco (Julca y Crespo, 1998). 


\section{Respuesta de variedades}

Para conocer la respuesta de las dos variedades de tabaco negro (HN1 y HN2) cultivadas en la zona de Tarapoto se delimitó un área experimental de aproximadamente una hectárea en campos comerciales de cada variedad y en cada caso se tomaron 200 plantas al azar en las que se evaluó la severidad por planta. El estudio se realizó entre los meses de enero y junio, tiempo durante el cual se tuvieron las variedades instaladas de forma simultánea en el campo.

\section{Efecto de la edad de la planta}

Para determinar el efecto de la edad de la planta en la severidad de la enfermedad se tomaron 50 plantas al azar en parcelas comerciales de tabaco de la variedad HN2 de diferentes edades. El estudio se realizó durante el tiempo que dura el cultivo (aproximadamente 90 días). Los datos fueron sometidos a un Análisis de Regresión Lineal simple.

\section{Efecto de la enfermedad en el rendimiento}

Para determinar el efecto de la edad de la planta en el rendimiento de tabaco negro var. HN2 se tomaron 200 plantas al azar en parcelas comerciales de tabaco de la variedad HN2 y se evaluó la severidad por planta, el peso fresco y peso seco de las hojas. El estudio se realizó durante el periodo del cultivo y los datos fueron sometidos a un Análisis de Regresión Lineal simple.

\section{Efecto de la densidad de plantación}

Se estudiaron tres cantidades diferentes de plantas por unidad de área (Tabla 1). El punto de referencia fue 33.300 plantas/ha. Esta es la densidad recomendada en las zonas productoras de tabaco negro en el Perú. El experimento se repitió dos veces (época seca y lluviosa) y se evaluó la severidad de la enfermedad a la cosecha (primer, tercer y quinto corte).
Tabla 1

Tratamientos para conocer efecto de la densidad de plantación sobre la "Cercosporiosis"

(Cercospora nicotianae) en Tabaco Negro var. HN2, Distrito de Juan Guerra, Tarapoto, Perú

\begin{tabular}{|c|c|c|c|}
\hline \multirow{2}{*}{ Tratamientos } & \multicolumn{2}{|c|}{ Distanciamiento (m) } & \multirow{2}{*}{$N^{\mathbf{o}}$ Plantas/ha } \\
\cline { 2 - 3 } & Entre plantas & Entre hileras & \\
\hline $\mathrm{D}_{1}$ & 1,00 & 0,30 & 33.300 \\
\hline $\mathrm{D}_{2}$ & 1,00 & 0,35 & 28.500 \\
\hline $\mathrm{D}_{3}$ & 1,00 & 0,40 & 25.000 \\
\hline
\end{tabular}

Se trabajó con un diseño de bloques completos al azar (DBCA), con cuatro repeticiones para cada tratamiento. Las unidades experimentales tuvieron tres hileras con 14 plantas cada una; pero las evaluaciones se hicieron en las 10 plantas de la hilera central.

\section{Efecto de la fertilización}

En este ensayo se trabajó con cinco tratamientos que consideró cuatro niveles de $\mathrm{N}-\mathrm{P}_{2} \mathrm{O} 5-\mathrm{K}_{2} \mathrm{O}$ - MgO, más un testigo sin fertilizar (Tabla 2). La cantidad total de fertilizantes se fraccionó en dos partes; la primera $(40 \%)$ se aplicó siete días después del trasplante, el restante (60\%) se aplicó quince días después. La fuente usada fue el fertilizante compuesto $12 \mathrm{~N}-12 \mathrm{P}_{2} \mathrm{O}_{5}-12 \mathrm{~K}_{2} \mathrm{O}-4 \mathrm{MgO}$. $\mathrm{El}$ experimento se repitió dos veces (época seca y lluviosa) y se evaluó la severidad de la enfermedad a la cosecha (primer, tercer y quinto corte).

Tabla 2

Tratamientos para conocer efecto de la fertilización sobre la "Cercosporiosis" (Cercospora nicotianae) en tabaco negro var. HN2 (33.300 plantas/ha), Distrito de Juan Guerra, Tarapoto, Perú

\begin{tabular}{|c|c|c|c|c|}
\hline Tratamientos & $\begin{array}{c}\mathbf{N} \\
(\mathbf{K g} / \mathbf{h a})\end{array}$ & $\begin{array}{c}\mathbf{P}_{\mathbf{2}} \mathbf{O}_{\mathbf{5}} \\
(\mathbf{K g} / \mathbf{h a})\end{array}$ & $\begin{array}{c}\mathbf{K}_{\mathbf{2}} \mathbf{O} \\
(\mathbf{K g} / \mathbf{h a})\end{array}$ & $\begin{array}{c}\mathbf{M g O} \\
(\mathbf{K g} / \mathbf{h a})\end{array}$ \\
\hline $\mathrm{F}_{0}$ & 0 & 0 & 0 & 0 \\
\hline $\mathrm{F}_{1}$ & 60 & 60 & 60 & 20 \\
\hline $\mathrm{F}_{2}$ & 90 & 90 & 90 & 30 \\
\hline $\mathrm{F}_{3}$ & 120 & 120 & 120 & 40 \\
\hline $\mathrm{F}_{4}$ & 150 & 150 & 150 & 50 \\
\hline
\end{tabular}


Se trabajó con un diseño de bloques completos al azar (DBCA), con cuatro repeticiones para cada tratamiento. Las unidades experimentales tuvieron tres surcos con 14 plantas cada uno, pero las evaluaciones se hicieron en las 10 plantas de la hilera central.

\section{Efecto del control de brotes axilares}

En este ensayo se trabajó con siete tratamientos, incluido un testigo sin control de brotes axilares (Tabla 3). Se evaluó la severidad de la enfermedad a la cosecha (tercer y quinto corte).

Tabla 3

Efecto del control de brotes axilares sobre la "Cercosporiosis" en tabaco negro, var. HN2. Tarapoto, Perú

\begin{tabular}{|c|c|}
\hline Tratamientos & Descripción \\
\hline $\mathrm{T} 1$ & Prowl $\left(5 \%_{\mathrm{o}}\right)$ \\
\hline $\mathrm{T} 2$ & Prowl $\left(10 \%_{\mathrm{o}}\right)$ \\
\hline $\mathrm{T} 3$ & Prowl $\left(15 \%_{\mathrm{o}}\right)$ \\
\hline $\mathrm{T} 4$ & Prowl $\left(20 \%_{\mathrm{o}}\right)$ \\
\hline $\mathrm{T} 5$ & FST7 $\left(37.5 \%_{\mathrm{o}}\right)$ \\
\hline $\mathrm{T} 6$ & Control Manual \\
\hline $\mathrm{T} 7$ & Sin control \\
\hline
\end{tabular}

Se trabajó con un diseño de bloques completos al azar (DBCA), con cuatro repeticiones para cada tratamiento. Las unidades experimentales tuvieron tres surcos con 14 plantas cada uno, pero las evaluaciones se hicieron en las 10 plantas del surco central.

\section{Efecto del control químico}

En este ensayo se trabajó con 13 tratamientos, incluido un testigo sin tratar (Tabla 4). El experimento se repitió dos veces (época seca y lluviosa) y se evaluó la severidad de la enfermedad a la cosecha (primer, tercer y quinto corte).

También en este caso se usó un diseño de bloques completos al azar (DBCA), con cuatro repeticiones para cada tratamiento. Las unidades experimentales tuvieron tres surcos con 14 plantas cada uno; pero las evaluaciones se hicieron en las 10 plantas del surco central.

\section{RESULTADOS Y DISCUSIÓN}

De manera general, los resultados de este estudio muestran el ataque importante de la "Cercosporiosis" (Cercospora nicotianae) en el cultivo de tabaco negro en la zona de Juan Guerra en Tarapoto, corroborando

Tabla 4

Tratamientos para el control químico de Cercospora nicotianae en tabaco negro, var. HN2. Tarapoto, Perú

\begin{tabular}{|c|c|c|c|c|c|c|c|c|c|c|}
\hline Tratamientos & \multicolumn{10}{|c|}{ Descripción } \\
\hline CQ0 & \multicolumn{10}{|c|}{ Testigo (sin control) } \\
\hline CQ1 & $\mathrm{TeC}$ & $\mathrm{MaA}$ & $\mathrm{TeC}$ & $\mathrm{MeA}$ & $\mathrm{TeC}$ & $\mathrm{MaA}$ & $\mathrm{TeC}$ & $\mathrm{MeA}$ & $\mathrm{TeC}$ & $\mathrm{MaA}$ \\
\hline CQ2 & $\mathrm{BeC}$ & $\mathrm{MaA}$ & $\mathrm{BeC}$ & $\mathrm{MeA}$ & $\mathrm{BeC}$ & $\mathrm{MaA}$ & $\mathrm{BeC}$ & $\mathrm{MeA}$ & $\mathrm{BeC}$ & $\mathrm{MaA}$ \\
\hline CQ3 & $\mathrm{MaA}$ & $\mathrm{TeC}$ & $\mathrm{MaA}$ & $\mathrm{BeC}$ & $\mathrm{MaA}$ & $\mathrm{TeC}$ & $\mathrm{MaA}$ & $\mathrm{BeC}$ & $\mathrm{MaA}$ & $\mathrm{TeC}$ \\
\hline CQ4 & $\mathrm{MeA}$ & $\mathrm{TeC}$ & $\mathrm{MeA}$ & $\mathrm{BeC}$ & $\mathrm{MeA}$ & $\mathrm{TeC}$ & $\mathrm{MeA}$ & $\mathrm{BeC}$ & $\mathrm{MeA}$ & $\mathrm{TeC}$ \\
\hline CQ5 & $\mathrm{TeCMaA}$ & $\mathrm{TeC} \mathrm{MeA}$ & $\mathrm{TeC} \mathrm{MaA}$ & $\mathrm{TeC} \mathrm{MeA}$ & $\mathrm{TeC} \mathrm{MaA}$ & $\mathrm{TeC} \mathrm{MeA}$ & $\mathrm{TeC} \mathrm{MaA}$ & $\mathrm{TeC} \mathrm{MeA}$ & $\mathrm{TeC} \mathrm{MaA}$ & $\mathrm{TeC} \mathrm{MeA}$ \\
\hline CQ6 & $\mathrm{BeC} \mathrm{MaA}$ & $\mathrm{BeC} \mathrm{MeA}$ & $\mathrm{BeC} \mathrm{MaA}$ & $\mathrm{BeC} \mathrm{MeA}$ & $\mathrm{BeC} \mathrm{MaA}$ & $\mathrm{BeC} \mathrm{MeA}$ & $\mathrm{BeC} \mathrm{MaA}$ & $\mathrm{BeC} \mathrm{MeA}$ & $\mathrm{BeC} \mathrm{MaA}$ & $\mathrm{BeC} \mathrm{MeA}$ \\
\hline CQ7 & $\mathrm{TeD}$ & $\mathrm{MaB}$ & $\mathrm{TeD}$ & $\mathrm{MeB}$ & $\mathrm{TeD}$ & $\mathrm{MaB}$ & $\mathrm{TeD}$ & $\mathrm{MeB}$ & $\mathrm{TeD}$ & $\mathrm{MaB}$ \\
\hline CQ8 & $\mathrm{BeD}$ & $\mathrm{MaB}$ & $\mathrm{BeD}$ & $\mathrm{MeB}$ & $\mathrm{BeD}$ & $\mathrm{MaB}$ & $\mathrm{BeD}$ & $\mathrm{MeB}$ & $\mathrm{BeD}$ & $\mathrm{MaB}$ \\
\hline $\mathrm{CQ9}$ & $\mathrm{MaB}$ & $\mathrm{TeD}$ & $\mathrm{MaB}$ & $\mathrm{BeD}$ & $\mathrm{MaB}$ & $\mathrm{TeD}$ & $\mathrm{MaB}$ & $\mathrm{BeD}$ & $\mathrm{MaB}$ & $\mathrm{TeD}$ \\
\hline CQ10 & $\mathrm{MeB}$ & $\mathrm{TeD}$ & $\mathrm{MeB}$ & $\mathrm{BeD}$ & $\mathrm{MeB}$ & $\mathrm{TeD}$ & $\mathrm{MeB}$ & $\mathrm{BeD}$ & $\mathrm{MeB}$ & $\mathrm{TeD}$ \\
\hline CQ11 & TeDMaB & $\mathrm{TeD} \mathrm{MeB}$ & TeDMaB & TeDMeB & TeDMaB & TeDMeB & TeDMaB & TeDMeB & TeDMaB & $\mathrm{TeDMeB}$ \\
\hline CQ12 & $\mathrm{BeDMaB}$ & $\mathrm{BeDMeB}$ & $\mathrm{BeDMaB}$ & $\mathrm{BeDMeB}$ & $\mathrm{BeDMaB}$ & $\mathrm{BeDMeB}$ & $\mathrm{BeDMaB}$ & $\mathrm{BeDMeB}$ & $\mathrm{BeDMaB}$ & $\mathrm{BeDMeB}$ \\
\hline $\begin{array}{l}\text { Fungicidas: } \\
\text { Dosis: }\end{array}$ & $\begin{array}{l}\text { Be: Benomyl } \\
\mathrm{A}=2.00 \% \text { o }\end{array}$ & & $\begin{array}{l}: \text { Mancozeb } \\
=2.50 \%\end{array}$ & $\begin{array}{l}\text { Me: Met } \\
\mathrm{C}=1.50\end{array}$ & & $\begin{array}{l}\text { Te: Tebucona } \\
D=2.00 \%\end{array}$ & & & & \\
\hline
\end{tabular}


que la enfermedad está muy generalizada en dicha zona, además de haber sido reportada en otras zonas productoras del Perú, como Tingo María y Satipo (Ames, 1968). El hongo aislado en medio Agar $\mathrm{V}-8$ se caracteriza por tener conidioforos (113 x 5 $\mu$ ) fasciculados, oscuros, septados, geniculados y con una cicatriz en el lugar donde esporulan; las conidias $(80 \times 4 \mu)$ son aciculares, hialinas, con nueve septas y una cicatriz oscura en la base (Julca y Crespo, 1998).

Esta enfermedad es una de las más importantes de este cultivo, causa pérdidas frecuentemente en los países tropicales (APS, 1991) y ataca a diferentes tipos de tabaco. Así tenemos que, en el tabaco Burley, las manchas causadas por este patógeno también son fuertes y afectan su rendimiento y calidad (Anjeneyulu et al., 1988), lo mismo ocurre en el tabaco rubio o Virginia (Crespo y Julca, 2005).

\section{ANÁLISIS DE LA METODOLOGÍA DE EVALUACIÓN}

Cuando se estudian las enfermedades de las plantas, es necesario calcular los daños que ocasionan los fitopatógenos, ya sea para estimar la intensidad de dichos daños o para conocer la respuesta a las diferentes medidas de control (Mont, 1993). Dentro de este aspecto, la incidencia y la severidad son dos parámetros que permiten evaluar una enfermedad. El mayor problema es medir la severidad; para ello se ha sugerido diseñar escalas, las que pueden ser descriptivas o diagramatizadas (FAO, 1985).

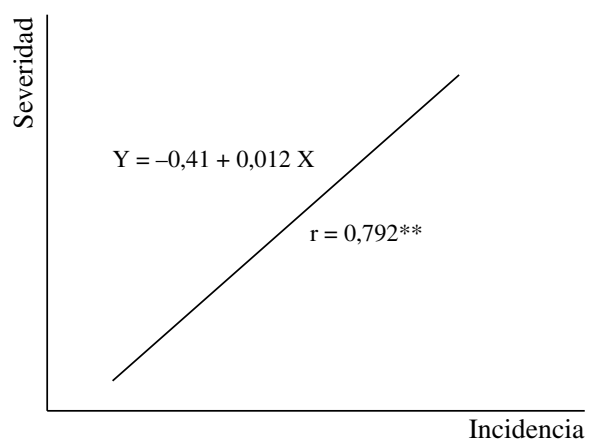

Figura 3. Línea de regresión y coeficiente de correlación (r) entre severidad $\left(\mathrm{S}_{\mathrm{p}}\right)$ e incidencia de Cercospora nicotianae en tabaco negro var. HN2. Localidad de Juan Guerra (Tarapoto).
Pero para que la evaluación de las enfermedades se realice de manera adecuada deben usarse metodologías sencillas y prácticas para que su uso no solamente sea entendido por los especialistas sino también por los productores, de tal forma que les permita obtener la información correcta para tomar las medidas de control que las circunstancias ameriten y en el momento preciso. En tabaco, para Cercospora nicotianae, se cuenta el número de manchas por hoja (Anjeneyulu et al., 1988); en maní se calcula el Índice Porcentual de la Enfermedad para Cercospora arachidicola (Chandra, et al., 1998); en remolacha azucarera reportan porcentaje de campos atacados y porcentaje de área foliar atacada para el caso de Cercospora betícola (Hermann et Menús, 1998).

En cultivos como el tabaco y otros, donde el objeto de cosecha son las hojas, la severidad es la variable más recomendable de evaluar, pues señala de manera más directa la importancia económica de la enfermedad. Además la severidad y la incidencia de la "Cercosporiosis" son dos variables que tienen una relación directa y el índice de correlación (r) obtenido es altamente significativo (Figura 3 ). En Costa Rica, para Cercospora coffeícola, se recomienda evaluar solamente la incidencia de la enfermedad debido a que la incidencia y la severidad también están altamente correlacionadas (Samayoa y Sánchez, 2000); pero en este caso el objeto de cosecha son los cerezos o frutos del café.

\section{EFECTO DE LA ÉPOCA DEL CULTIVO}

La incidencia y severidad de las enfermedades puede variar de una localidad a otra y de una estación a otra. Por ejemplo, Cercospora arachidicola es más importante y severa en Meghalaya, comparada con otras zonas productoras de maní en la India (Chandra et al., 1998), en Francia, también se ha reportado una gran variabilidad de la incidencia de manchas foliares que atacan a la remolacha azucarera (Hermann et Menús, 1998). En nuestro caso la "Cercosporiosis" adquiere características alarmantes cuando existe mucha humedad (Ames, 1968). En Tarapoto, la severidad de C. nicotianae fue 53,8\% más alta en la época de mayor precipitación que en la denominada época seca (Figura 4). Esto debido a que en presencia de lluvias las lesiones causadas por el hongo crecen rápidamente y el hongo empieza a esporular ocasionando infecciones adicionales (Ames, 1968). 


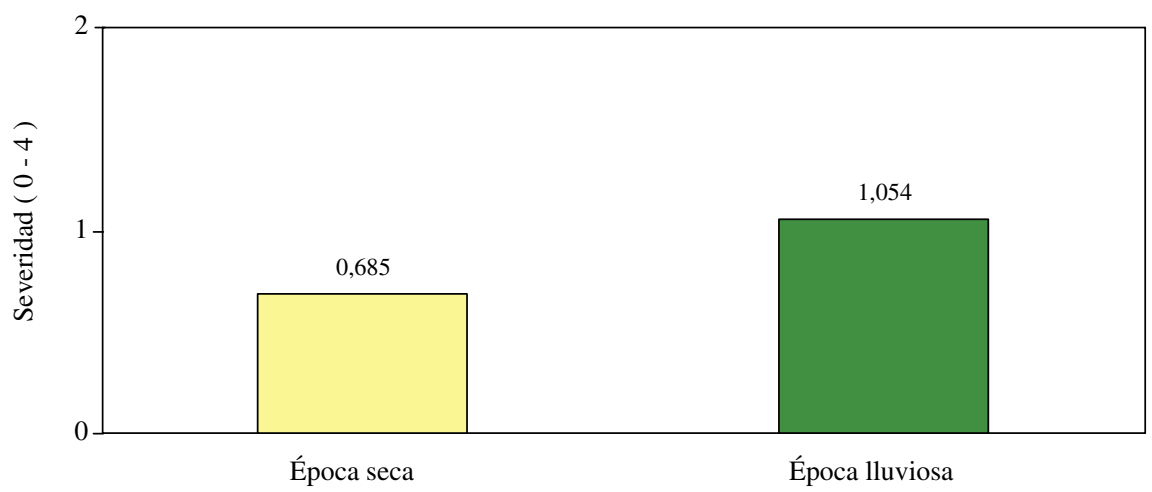

Figura 4. Efecto de la época del cultivo tabaco negro, var. HN2 en la severidad $\left(\mathrm{S}_{\mathrm{p}}\right)$ de Cercospora nicotianae. Tarapoto. Perú.

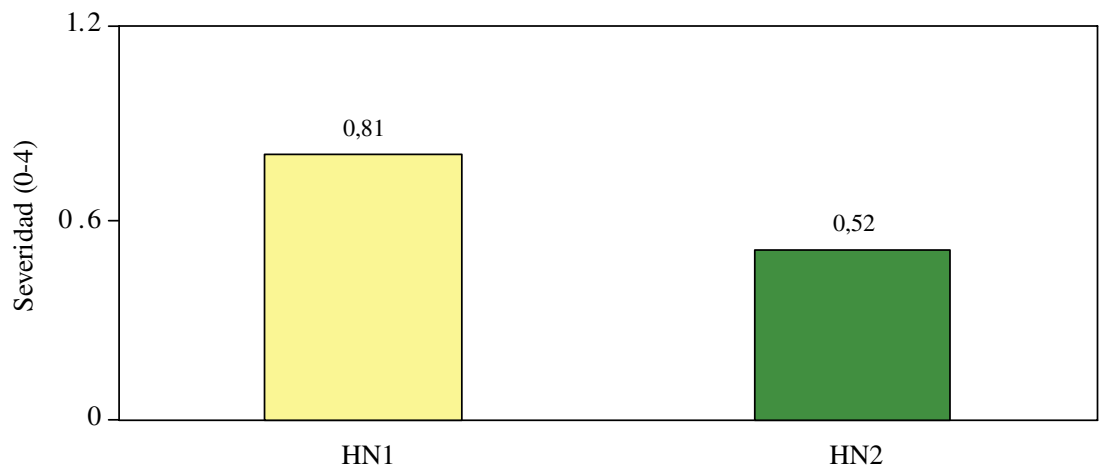

Figura 5. Respuesta de dos variedades de tabaco negro al ataque de Cercospora nicotianae en la localidad de Juan Guerra. Tarapoto. Perú.

\section{EFECTO DE LAS VARIEDADES}

El uso de variedades resistentes o tolerantes a las enfermedades es una opción importante para el manejo de las enfermedades en los cultivos agrícolas (Mont, 1993). En nuestro país se siembran diversas variedades de tabaco negro (HN1, HN2, Pinar del río, Pelo de oro, Corojo y Kentucky); todas han mostrado susceptiblidad a la "Cercosporiosis" (Echeandía, 2007) y la decisión de sembrar una $\mathrm{u}$ otra variedad depende principalmente de las preferencias del mercado.

En nuestro estudio, la variedad HN2 tuvo una severidad 35,8\% menor que el valor reportado en la variedad HN1 (Figura 5), resultado interesante si consideramos que la primera es la más aceptada comercialmente. Según Echeandía (2007), la HN2 es una variedad de la que se puede obtener capa (aunque en baja proporción), capote y tripa. Se caracteriza porque al despunte tiene una altura aproximada de $1,70 \mathrm{~m}$ y puede llegar a producir hasta 26 hojas (largo: 60 a $65 \mathrm{~cm}$ y ancho: $30 \mathrm{~cm}$ ) que son de grano abierto y mucha elasticidad, la cosecha empieza 50 días después del trasplante y termina 30 días más tarde.

En los últimos años, diversos investigadores buscan fuentes de resistencia a C. nicotianae e intentan explicar los mecanismos que la regulan. Así tenemos que en la India se han evaluado cinco cultivares de Nicotiana rustica y se encontró que Rustica Swabi y Naswari fueron resistentes al ataque del hongo (Ahmed and Yaqub, 1994). Goy et al. (1993) encontraron que la escopolina y la escopoletina son dos compuestos fenoles que se encuentran en gran cantidad en las hojas de un tabaco híbrido (N. glutinosa x N. debneyi), característica que le da una alta resistencia al ataque de C. nicotaianae y otros hongos. Mientras que Dixon et al. (1996), trabajando con plantas transgénicas de tabaco, encontraron que la sobreexpresión de la 

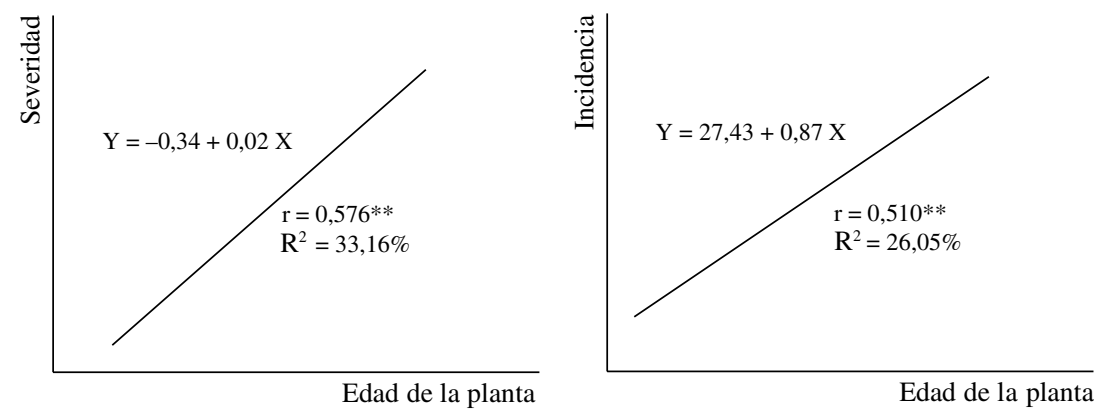

Figura 6. Líneas de regresión, coeficientes de correlación (r) y determinación (R2) para edad de planta con severidad (izquierda) e incidencia (derecha) de la "Cercosporiosis" en tabaco negro var. HN2. Tarapoto. Perú.

quitinasa y la glucanasa incrementa la resistencia a C. nicotianae y Rhizoctonia solani.

\section{Efecto de la edad de planta}

Algunos autores sostienen que la "Cercosporiosis" se hace más notoria conforme avanza la edad de la planta de tabaco. C. nicotianae es considerado un patógeno débil, ataca solamente tejido fisiológicamente en decadencia (Ames, 1968). En nuestro estudio encontramos que tanto la incidencia como la severidad están correlacionadas de manera directa con la edad de la planta. Esta variable explica aproximadamente un tercio del nivel que alcanza la enfermedad (Figura 6). Resultados similares se reportaron para el caso del tabaco Burley en la India (Anjeneyulu et al., 1988). Es que las hojas maduras son más susceptibles que las hojas tiernas (Ames, 1968). Por ello la importancia de realizar una cosecha oportuna. La cosecha de hojas se debe realizar cuando éstas reúnan los criterios de madurez; debe evitarse que las hojas envejezcan en la planta (Crespo y Julca, 2005).

\section{Efecto de la "Cercosporiosis" en el rendimiento de tabaco}

La correlación entre la severidad de Cercospora nicotianae y el rendimiento de tabaco negro var. HN2 siempre fue negativa y los valores que alcanzó el índice de correlación (r) tanto para peso fresco como para peso seco fueron altamente significativos (Figura 7). El coeficiente de determinación (R2) muestra que la severidad $\left(S_{p}\right)$ explica el rendimiento en un porcentaje prácticamente similar tanto para el peso fresco como para el peso seco de este tipo de tabaco; pero este valor disminuye con el paso del tiempo. Así tenemos que la severidad calculada antes del primer corte o cosecha de hojas (1C) explica más del 50\% del peso fresco; pero solamente un tercio al momento de la última cosecha de hojas (5C), la misma tendencia se observa para el peso seco de hojas (Figura 7).

Si bien los modelos de regresión desarrollados en este estudio muestra tendencias interesantes, es recomendable que para otras zonas productoras de tabaco estos se tomen solamente como referencia y desarrollen sus propios modelos de regresión. Esto debido a que una correlación solamente es válida para las condiciones geográficas y climáticas en la que ha sido establecida (Combres, 1979 ).

Por otra parte, los resultados sugieren la importancia de tomar medidas de control lo más temprano posible para evitar el efecto negativo que tiene la enfermedad sobre el rendimiento de tabaco negro en la localidad de Juan Guerra en la zona nororiental del Perú. C. nicotianae reduce fuertemente el rendimiento y calidad de otros tipos de tabaco, como es el caso del tabaco burley, y es considerado un factor limitante para su producción (Anjeneyulu et al., 1988). C. zeae-maydis disminuye seriamente la producción de grano en maíz (Ward et al., 1996) у Cercospora betícola, en remolacha azucarera, disminuye la cantidad y la calidad de la cosecha (Hermann et Menús, 1999).

\section{Efecto de la densidad de plantación}

La densidad o marco de plantación se refiere a la instalación de un número adecuado de plantas para un área específica de tal manera que permita una cosecha óptima en calidad y cantidad (Urbano, 2002). La cantidad de plantas por unidad de área y su disposición en el campo permitirá un mayor apro- 

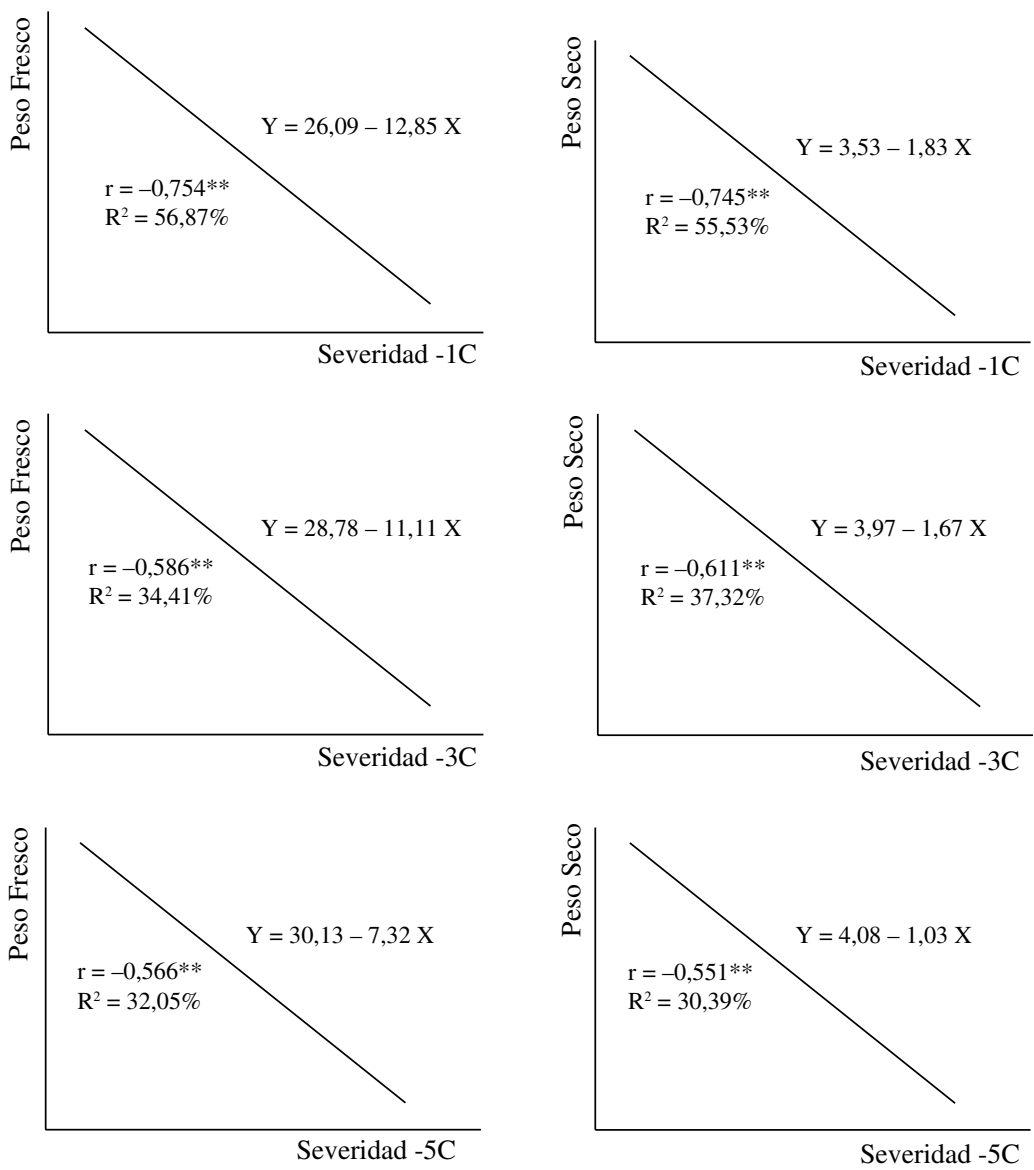

Figura 7. Líneas de regresión, coeficientes de correlación (r) y de determinación (R2) para estimar efecto de Cercospora nicotianae en el rendimiento de tabaco negro, var. HN2. Tarapoto, Perú.

vechamiento de la luz, mejor absorción de nutrientes y la no competencia entre plantas (Crespo y Julca, 2005). En este estudio, la densidad de plantación no tuvo efecto significativo sobre la "cercosporiosis" en ningún momento de la evaluación; pero los resultados mostraron que el valor más alto de severidad correspondió siempre al tratamiento con un mayor número de plantas/ha $\left(\mathrm{D}_{1}\right)$, tal como se observa en la Tabla 5.

Los resultados sugieren que los niveles de plantación evaluados en este estudio no han sido lo suficientemente diferentes uno del otro como para generar cambios en el microclima dentro de cada una de las parcelas de tabaco, aspecto que favorecería o no la presencia del patógeno. En Costa Rica, Polanco et al. (1982) tampoco encontraron diferencias en la incidencia de esta enfermedad cuando compararon tres distancias entre hileras $(0,40,0,45$ y $0,50 \mathrm{~m})$ y tres distancias entre plantas $(1,00,1,30$ y $1,60 \mathrm{~m})$ en tabaco var. NC-79.

En otros cultivos se ha reportado una relación directa entre el desarrollo de la enfermedad y la densidad de plantación. Así tenemos que en espárrago la roya (Puccinia asparagi) presentó la más alta severidad en las parcelas con una mayor densidad de plantas (Apaza, 1997); lo mismo ocurrió en cacao (Theobroma cacao) para el caso la "escoba de brujas", una enfermedad causada por el hongo Crinipellis perniciosa (Dias et al., 2000). 
Tabla 5

Efecto de la densidad de plantación en la "Cercosporiosis" en tabaco negro, var. HN2. Tarapoto, Perú (*).

\begin{tabular}{|c|c|c|c|}
\hline \multirow{2}{*}{ Tratamientos } & \multicolumn{3}{|c|}{ Severidad $\left(\mathbf{S}_{\mathbf{p}}\right)$ de Cercospora nicotianae } \\
\cline { 2 - 4 } & Primer Corte & Tercer Corte & Quinto Corte \\
\hline $\mathrm{D}_{1}$ & $0,451 \mathrm{a}$ & $0,848 \mathrm{a}$ & $1,345 \mathrm{a}$ \\
\hline $\mathrm{D}_{2}$ & $0,277 \mathrm{a}$ & $0,756 \mathrm{a}$ & $1,320 \mathrm{a}$ \\
\hline $\mathrm{D}_{3}$ & $0,326 \mathrm{a}$ & $0,809 \mathrm{a}$ & $1,075 \mathrm{a}$ \\
\hline
\end{tabular}

(*): Resultados son promedios de dos ensayos realizados en épocas diferentes (seca y lluviosa).

\section{Efecto de la fertilización}

Para los dogmáticos una planta bien nutrida no se enferma ni es atacada por las plagas (Brenes, 2003). Lamentablemente el desarrollo de las enfermedades en las plantas cultivadas no es un fenómeno simple de explicar, por lo que la nutrición mineral puede aumentar o disminuir la resistencia o tolerancia de las plantas a las plagas y enfermedades (Gárate y Bonilla, 2001). En el caso del "Ojo de rana", $\boldsymbol{l a}$ fertilización mineral no generó diferencias estadísticas sobre la severidad en ningún momento de la evaluación; pero los resultados mostraron que el valor más alto correspondió siempre al tratamiento con un mayor nivel de fertilización $\left(\mathrm{F}_{4}\right)$. Esto fue más notorio en el quinto corte, donde la severidad correspondiente al $\mathrm{F}_{4}$ fue $37,6 \%$ superior al tratamiento testigo (Tabla 6).

Ames (1968) señala que para el manejo de $C$. nicotianae debe evitarse la sobrefertilización con nitrógeno, ya que aumentando la cantidad de $\mathrm{N}$ disponible también aumenta la susceptibilidad al ataque de este hongo. Pero si bien se ha señalado al $\mathrm{N}$ y K como elementos importantes que afectan la resistencia de los cultivos a las enfermedades (Gárate y Bonilla, 2001), esto no significa que el aumento de alguno de estos elementos, o de ambos, otorga siempre una mayor resistencia a la planta, porque la presencia de una enfermedad también depende del patógeno involucrado. Según Gárate y Bonilla (2001), una mayor concentración de K en la hoja aumenta la resistencia tanto a parásitos facultativos (p.e. Fusarium) como a obligatorios (p.e. Puccinia); en cambio, un incremento de $\mathrm{N}$ aumenta la resistencia a parásitos facultativos, pero disminuye la resistencia a los parásitos obligatorios.
Tabla 6

Efecto de fertilización sobre la "Cercosporiosis" en tabaco negro, var. HN2. Tarapoto, Perú (*)

\begin{tabular}{|c|c|c|c|}
\hline \multirow{2}{*}{ Tratamientos } & \multicolumn{3}{|c|}{ Severidad $\left(\mathbf{S}_{\mathbf{p}}\right)$ de Cercospora nicotianae } \\
\cline { 2 - 4 } & Primer Corte & Tercer Corte & Quinto Corte \\
\hline $\mathrm{F}_{0}$ & $0,45 \mathrm{a}$ & $0,68 \mathrm{a}$ & $1,41 \mathrm{a}$ \\
\hline $\mathrm{F}_{1}$ & $0,46 \mathrm{a}$ & $0,72 \mathrm{a}$ & $1,57 \mathrm{a}$ \\
\hline $\mathrm{F}_{2}$ & $0,51 \mathrm{a}$ & $0,74 \mathrm{a}$ & $1,55 \mathrm{a}$ \\
\hline $\mathrm{F}_{3}$ & $0,45 \mathrm{a}$ & $0,63 \mathrm{a}$ & $1,50 \mathrm{a}$ \\
\hline $\mathrm{F}_{4}$ & $0,53 \mathrm{a}$ & $0,75 \mathrm{a}$ & $1,94 \mathrm{a}$ \\
\hline
\end{tabular}

(*): Resultados son promedios de dos ensayos realizados en épocas diferentes (seca y lluviosa)

\section{Efecto del control de brotes axilares}

El control de brotes axilares en tabaco se puede hacer manualmente o usando productos químicos. De no ser controlados estos crecen a expensas de las hojas y afectan el peso y la composición química de las mismas (Crespo y Julca, 2005). En Tarapoto, cuando se evaluaron diversos tratamientos para el control de estos brotes en tabaco negro var. HN2, la severidad del "ojo de rana" siempre fue menor en las plantas donde el control fue manual, efecto que fue significativo en el quinto corte (Tabla 7). Cuando las plantas sufren lesiones, éstas acumulan ácido jasmónico y jasmonato de metilo, compuestos asociados con la resistencia al ataque de insectos y patógenos (Zacarías y Lafuente, 2000).

\section{Tabla 7}

Efecto del control de brotes axilares sobre la "Cercosporiosis" en tabaco negro, var. HN2. Tarapoto, Perú.

\begin{tabular}{|l|c|c|}
\hline \multirow{2}{*}{ Tratamientos } & \multicolumn{2}{|c|}{$\begin{array}{c}\text { Severidad }\left(\mathbf{S}_{\mathbf{p}} \text { ) de Cercospora }\right. \\
\text { nicotianae }\end{array}$} \\
\cline { 2 - 3 } & Tercer Corte & Quinto Corte \\
\hline Prowl - 5\%o & $0,792 \mathrm{a}$ & $1,171 \mathrm{a}$ \\
\hline Prowl - 10\%o & $0,753 \mathrm{a}$ & $0,982 \mathrm{ab}$ \\
\hline Prow1 - 15\%o & $0,642 \mathrm{a}$ & $0,822 \mathrm{ab}$ \\
\hline Prowl - 20\%o & $0,614 \mathrm{a}$ & $0,926 \mathrm{ab}$ \\
\hline FST7 - 37.5\%o & $0,697 \mathrm{a}$ & $0,832 \mathrm{ab}$ \\
\hline Control Manual & $0,597 \mathrm{a}$ & $0,752 \mathrm{~b}$ \\
\hline Sin control & $0,631 \mathrm{a}$ & $0,936 \mathrm{ab}$ \\
\hline
\end{tabular}




\section{Efecto del control químico}

Para el control de la "cercosporiosis" en tabaco, las experiencias han estado dirigidas generalmente al uso de fungicidas químicos (Ames, 1968; Yaringaño et al., 1975; Chadwani and Lal, 1979; Esquivel y Raby, 1979; Polanco et al., 1982; Anjeneyulu et al., 1988; Ahmed y Shafique, 1994). En este ensayo, todos los tratamientos evaluados tuvieron una severidad menor que en la parcela testigo $\left(\mathrm{CQ}_{0}\right)$, pero estas diferencias no fueron estadísticamente significativas durante las dos primeras evaluaciones; sí en las hojas del último corte o cosecha (Tabla 7).

Los cuatro productos evaluados en este trabajo ya habían demostrado su efecto negativo sobre C. nicotianae en un ensayo de laboratorio (Julca y Crespo, 1998). En campo, las mayores experiencias para el control de este hongo corresponden al Benomyl que se recomienda en el cultivo de tabaco en América Central (APS, 1991) y en la India (Parvatha Reddy, 1978; Ahmad y Shafique, 1994). El Mancozeb también está recomendado para el control de $C$. nicotianae en la India (Chandawani y Ranjilal, 1979; Patel et al., 1991), además de C. capsici y C. fabae en ají y haba, respectivamente (anónimo, 1996). En mezcla con Carbendazin, controló muy bien $C$. arachidicola en maní (Chandra et al., 1998).

El Metiran se sugiere para el control de Cercospora spp. en el cultivo de tabaco (anónimo, 1996); resultados semejantes se obtuvieron con Thiran, en el cultivo de soya contra C. kikuchii (Lopes y De Camargo, 1997). Mientras que el Tebuconazole se usa contra C. musae en banano, Cercospora spp. en maní (Bayer, 1996) y C. arachidicola en maní (Lopes, et al., 1997).

Otro aspecto importante que muestra la Tabla 7 es que aquellos tratamientos donde hay una mezcla de fungicidas sistémicos y de contacto $\left(\mathrm{CQ}_{5}, \mathrm{CQ}_{6}, \mathrm{CQ}_{11}\right.$ y $\mathrm{CQ}_{12}$,) son los que mejor controlan la enfermedad y presentan los menores valores de severidad. Experiencias similares han sido reportadas por otros investigadores en Perú. Por ejemplo, al evaluarse diferentes estrategias de control químico para Puccinia asparagi en el cultivo del espárrago, se encontró que los mejores resultados correspondieron a la mezcla de un fungicida sistémico más uno de contacto (Apaza, 1997).

La combinación de dos grupos de fungicidas con diferente modo de acción no solamente otorga un buen control de la enfermedad y un mayor periodo de protección al cultivo, sino también disminuye
Tabla 7

Efecto del control químico sobre la "Cercosporiosis" en tabaco negro, var. HN2. Tarapoto, Perú (*).

\begin{tabular}{|l|c|c|c|}
\hline \multirow{2}{*}{ Tratamientos } & \multicolumn{3}{|c|}{ Severidad $\left(\mathbf{S}_{\mathbf{p}}\right)$ de Cercospora nicotianae } \\
\cline { 2 - 4 } & Primer Corte & Tercer Corte & Quinto Corte \\
\hline $\mathrm{CQ}_{0}$ & $0,93 \mathrm{a}$ & $1,24 \mathrm{a}$ & $2,00 \mathrm{a}$ \\
\hline $\mathrm{CQ}_{1}$ & $0,56 \mathrm{a}$ & $0,79 \mathrm{a}$ & $1,48 \mathrm{ab}$ \\
\hline $\mathrm{CQ}_{2}$ & $0,53 \mathrm{a}$ & $0,90 \mathrm{a}$ & $1,31 \mathrm{~b}$ \\
\hline $\mathrm{CQ}_{3}$ & $0,48 \mathrm{a}$ & $0,77 \mathrm{a}$ & $1,42 \mathrm{ab}$ \\
\hline $\mathrm{CQ}_{4}$ & $0,51 \mathrm{a}$ & $0,77 \mathrm{a}$ & $1,35 \mathrm{~b}$ \\
\hline $\mathrm{CQ}_{5}$ & $0,35 \mathrm{a}$ & $0,68 \mathrm{a}$ & $1,05 \mathrm{~b}$ \\
\hline $\mathrm{CQ}_{6}$ & $0,46 \mathrm{a}$ & $0,71 \mathrm{a}$ & $1,04 \mathrm{~b}$ \\
\hline $\mathrm{CQ}_{7}$ & $0,44 \mathrm{a}$ & $0,81 \mathrm{a}$ & $1,35 \mathrm{~b}$ \\
\hline $\mathrm{CQ}_{8}$ & $0,50 \mathrm{a}$ & $0,83 \mathrm{a}$ & $1,27 \mathrm{~b}$ \\
\hline $\mathrm{CQ}_{9}$ & $0,50 \mathrm{a}$ & $0,85 \mathrm{a}$ & $1,42 \mathrm{ab}$ \\
\hline $\mathrm{CQ}_{10}$ & $0,44 \mathrm{a}$ & $0,77 \mathrm{a}$ & $1,33 \mathrm{~b}$ \\
\hline $\mathrm{CQ}_{11}$ & $0,23 \mathrm{a}$ & $0,64 \mathrm{a}$ & $1,10 \mathrm{~b}$ \\
\hline $\mathrm{CQ}_{13}$ & $0,40 \mathrm{a}$ & $0,59 \mathrm{a}$ & $1,00 \mathrm{~b}$ \\
\hline
\end{tabular}

(*): Resultados son promedios de dos ensayos realizados en épocas diferentes (seca y lluviosa)

el riesgo de la aparición de resistencia del hongo a estos productos (Ward et al., 1997), problema que se ha planteado para el caso de Benomyl usado contra C. nicotianae en el cultivo de tabaco (APS, 1991), C. arachidicola en maní (Rao et al., 1993) y con $C$. zea-maydis, en maíz (Ward et al., 1997).

\section{CONCLUSIONES}

- La severidad y la incidencia de la "Cercosporiosis" estuvieron altamente correlacionadas.

- La severidad aumenta con la edad de la planta de tabaco.

- La severidad fue menor en la variedad HN2.

- La severidad fue mayor en la época lluviosa.

- La densidad de plantación no afectó significativamente la severidad de la enfermedad.

- La fertilización no afectó significativamente la severidad de la enfermedad.

- La severidad fue significativamente menor cuando el control de brotes axilares se realizó manualmente.

- La aplicación de fungicidas sí controló significativamente la enfermedad; los mejores resultados estuvieron asociados con la mezcla de fungicidas de contacto y sistémicos. 


\section{AGRADECIMIENTOS}

Los autores quieren dejar constancia de su agradecimiento a la empresa Tabacos del Perú S.A. (TAPESA), por su apoyo para la realización de este trabajo, pero de manera especial a los Ing. Arturo
Rubio y Fernando Echeandía, Gerente Técnico y Jefe de Zona en Tarapoto, respectivamente. También al Biol. Juan Flores y Luis Gutiérrez (UNALM), por su apoyo para el montaje de las muestras y fotografías.

\section{LITERATURA CITADA}

AMES, T. 1968. Enfermedades del tabaco. En: Curso de capacitación profesional en tabaco. Convenio CONATA-UNALM. Lima. pp: 46-87.

ANJENEYULU, C.; NAGARAJAN, K. AND RAMJILAL. 1988. Evaluation of fungicides against frog-eye spot (Cercospora nicotianae) disease in transplanted burley tobacco. Indian Phytopath. 41: 233-235.

AMERICAN PHYTOPATHOLOGICAL SOCIETY (APS). 1991. Compendium of tobacco diseases. Ed. H.D. Shew and G.B. Lucas. Minnesota. USA. 68 pp.

AHMAD, I. y YAQUB, M. 1994. Field evaluation of rustica tobacco germoplasm for resistance against frog-eye disease caused by Cercospora nicotianae. Pakistan Journal of Phytopathology 6: 63-64.

AHMED, I. y SHAFIQUE, M. 1994. Fungicidal control of frog-eye disease of tobacco (Nicotiana rustica) caused by Cercospora nicotianae. Pakistan Journal of Phytopathology 6: 61-62.

APAZA, W. 1997. Control químico de la roya del espárrago. Fitopatología 32: 43-51.

ANÓNIMO. 1996. Vademecum Agrario. Ediprensa. Lima. Perú. 118 pp.

BAYER. 1996. Folicur Información Técnica. Lima. Perú. $28 \mathrm{pp}$.

BRENES, L. 2003. Producción orgánica: algunas limitaciones que enfrentan los pequeños productores. Manejo Integrado de Plagas y Agroecología 70: 7-18.

COMBRES, J. C. 1979. Contribution a etude de l'action du climat sur les principales composantes de la production. Reunion Anualle IRFA. Documento interne $\mathrm{N}^{\circ} 60$.

CISNEROS, F. 1980. Principios del control de plagas. Editorial Gráfica Pacific Press. Lima. 189 pp.

CRESPO, R. y JULCA, A. 2005. Manual del cultivo de tabaco rubio. UNALM - INIA. Lima. Perú. 145 pp.

CHANDWANI, G.H. y RAMJILAL. 1979. Evaluation of some systemic fungicides against frog-eye spot in burley tobacco. Pesticides 13: 40 y 44.

CHANDRA, S.; KUMAR, S. and SINGH, A.K. 1998. Management of Cercospora leaf spot of groundnut (Arachys hypogea $\mathrm{L}$.) with a single fungicidal spray. Internacional Journal of Pest Management. 44:135-137.

DIXON, R.; SEWALT, V.; HOWLES, P. and LAMB. C. 1996. Genetic manipulation of the phenylpropanoid pathway in transgenic tobacco: new fundamental insigths and prospects for crop improvement. Biotechnology and Biotechnology Equipment 4: 45-51.

DIAS, L.A.S.; SANTOS, M. M.; SANTOS, O.S.; ALMEIDA, C.M.V.C.; CRUZ, C.D. AND CARNEIRO, P.C.S. 2000. Effect of plantig density on yield and incidente of witches

broom disease in a young plantation of Irbid cacao tress. Exp. Agric. 36: 501-508.

ESQUIVEL, E. y RABY, C. 1979. Control químico del ojo de sapo del tabaco (Cercospora nicotianae Ell \& Ev.) en la república de Panamá. Resúmenes de los trabajos presentados en el 1er. Congreso Latinoamericano de Fitopatología (ALF, APS, AVS).Sección Control y Miscelánea. 4-9 de noviembre. Maracaibo. Venezuela.

ECHEANDÍA, F. y OCAMPO, F. 2002. Cultivo del tabaco habano bajo sistema de riego por goteo. TAPESA-HOPETA. Lima. 113 pp.

ECHEANDÍA, F. 2007. Variedades de tabaco negro en Tarapoto. $1 \mathrm{pp}$ (no publicado).

FAO. 1985. Manual para patólogos vegetales. Lima. 438 pp.

GOY, P.A.; SIGNER, H.; REIST, R.; AICHHOLZ, R.; BLUM W.; SCHMIDT, E. and KESSMANN, H. 1993. Accumulation of scopoletin is associated with the high disease resistance of the hybrid Nicotiana glutinosa $\mathrm{x}$ Nicotiana debneyi. Planta 191: 200-2006.

GÁRATE, A. y BONILLA, I. 2001. Nutrición mineral y producción vegetal. En: Fundamentos de Fisiología Vegetal. Azcón-Bieto y Talón (Eds.). McGraw-Hill/Interamericana de España, S.A.U.-Universitat de Barcelona. Madrid. pp: 113-130.

HERMANN, O. ET MENÚS, P. 1998. Incidence des maladies foliares cryptogamiques et rentabilite du traitement fongicide. Le Betteravier. Février. pp: 51-52.

HERMANN, O. ET MENÚS, P. 1999. Importante des maladies foliares cryptogamiques et rentabilite du traitement fongicide et betterave. Le Betteravier. Julio-agosto. pp: 12-14.

JULCA, A. y CRESPO, R. 1998. La "Cercosporiosis" del tabaco negro en Tarapoto; diagnóstico, propuesta para su evaluación y pruebas de laboratorio para su control. Informe Final. UNALM. Departamento de Fitotecnia. Lima. 16 pp. (no publicado).

LATORRE, B. 1989. Fungicidas y Nematicidas. Universidad Católica de Chile. 215 pp.

LOPES, M. E. et al. 1997. Controle químico das doencas foliares do amendoim (Arachis hypogea L.). Rev. de Agricultura, Piracicaba, V.72, fasc. 1: 69-84.

LOPES, M.E. y B. DE CAMARGO. 1997. Eficiencia de fungicides no controle de fungos en sementes de soja (Glycine max (L.) Merrill).Rev. de Agricultura, Piracicaba, V.72, fasc. 1:85-98

MONT, R. 1993. Principios del control de enfermedades de las plantas. Universidad Nacional Agraria La Molina. Lima. Perú. 287 pp.

PARVATHA REDDY, P. 1978. Chemical control of frog-eye leaf spot disease of flue cured tobacco in Karnataka. Pesticides. December. pp: 31-32. 
POLANCO, D.; VARGAS, E. y MORERA, F. 1982. Combate integral de Cercospora nicotianae en tabaco, clase estufado en la zona de Parrita, Costa Rica. Resúmenes de V Congreso Agronómico Nacional (Sección Fitopatología). Julio. San José. Costa Rica.

PATEL, B.N.; J.B. PATEL and D.G. TILVA. 1991. Management of frog eye spot disease in bidi tobacco nursery by fungicides. Indian Phytopathology. 44: 399-401.

REDDY, T.S.N.; K. NAGARAJAN and RAMJILAL. 1992. Effect of fungicides on the control of frog-eye spot disease (Cercospora nicotianae) of Burley tobacco. Tob. Res. 18: 159-161.

RAO, P.V.S.; RENARD, J.L.; WALIYAR, F.; MCDONALD, D. and SCHILLING, R. 1993. Variation in symptons of Cercospora arachidicola isolates on some groundnut genotypes. Oleagineux 48: 243-250.

SAMAYOA J. y SÁNCHEZ V. 2000. Enfermedades foliares en café orgánico y convencional. Manejo Integrado de Plagas 58: 9-19.
URBANO, P. 2002. Fitotecnia, ingeniería de la producción vegetal. Mundi-Prensa. Madrid. $528 \mathrm{pp}$.

WARD, J.M.J.; M.D. LAING y D.C. NOWELL. 1997. Chemical control of maize grey leaf spot. Crop protection. 16 (3): 265-271.

WARD, M.J.; HOHLS, T. LAING, M.D. and RIJKENBERG, F.H.J. 199. Fungicide responses of maize hybrids to greay leaf spot. Europan Journal of Plant Pathology.102: 765-771.

YARINGAÑO, V.; VALLES, C. y VAN DE MEER, F. 1975. Control químico del ojo de sapo del tabaco negro (Cercospora nicotianae) Ell \& Ef. En almácigos y plantaciones. Resúmenes de los trabajos presentados en el IV Congreso Peruano de Fitopatología. 6-9 de julio. Chiclayo.

ZACARÍAS, L. y LAFUENTE, M. 2001. Etileno, ácido absícico y otros reguladores del desarrollo. En: Fundamentos de Fisiología Vegetal. Azcón-Bieto y Talón (Eds.). McGraw-Hill/Interamericana de España, S.A.U. - Universitat de Barcelona. Madrid. pp: 361-375. 
